\title{
Dodecafluoropentane Emulsion (DDFPe) Decreases Stroke Size and Improves Neurological Scores in a Permanent Occlusion Rat Stroke Model
}

\author{
A.T. Brown, M.C. Arthur, J.S. Nix, J.A. Montgomery, R.D. Skinner, P.K. Roberson, Michael \\ Borrelli and W.C. Culp*
}

Department of Radiology, UAMS, 4300 W. Markham St. Little Rock, AR, 72205 USA

\begin{abstract}
Background: Dodecafluoropentane emulsion (DDFPe), given IV one hour after stroke, has been shown to greatly reduce the percent stroke volume $(\% \mathrm{SV})$ in rabbits. With repeated doses its effect continued for 24 hours.

Purpose: Test DDFPe as neuroprotective agent in permanent occlusion rat stroke models in Sprague Dawley (SD) and Spontaneously Hypertensive Rats (SHR) measuring both \%SV and neurological assessment scores (NAS).

Methods: The male rats received either saline (control), or one or four doses (1x or 4x) of DDFPe $(0.6 \mathrm{ml} / \mathrm{kg} \mathrm{IV)} \mathrm{one} \mathrm{hour}$ post stroke. Treatment groups were SD $(n=26)$ (control, $1 \mathrm{x}$ and $4 \mathrm{x} ; \mathrm{n}=12,7$ and 7 ) and SHR $(\mathrm{n}=14)$ (control, 1x and 4x; $n=7,3$ and 4 ). The $4 x$ doses were given at 1.5 hour intervals. At six hours post stroke, the rats received a NAS using standard tests for balance, reflexes, and motor performance. Then rats were euthanized and brains removed for TTC evaluation of $\% \mathrm{SV}$.

Results: For $\% \mathrm{SV}$ analysis strain differences were not significant therefore strains were combined. DDFPe significantly decreased \%SV in $1 \mathrm{x}$ and $4 \mathrm{xDDFPe}$ groups compared to control groups $(2.59 \pm 1.81$ and $0.98 \pm 0.88 v$ s. $9.24 \pm 6.06, \mathrm{p} \leq 0.001$ each; $\mathrm{p} \leq 0.0001$ for the overall test for treatment effect). The $1 \mathrm{x}$ versus $4 \mathrm{xDDFPe}$ groups were not significantly different $(\mathrm{p}=0.40)$. In NAS analysis both strains showed significant improvement with 4xDDFPe therapy vs. controls, (SD: $5.00 \pm 2.45$ vs. $9.36 \pm 3.56, \mathrm{p}=0.01$; SHR: $7.75 \pm 4.43$ vs. $12.14 \pm 3.08, \mathrm{p}=0.05)$. Differences between the 1x DDFPe group and controls were not significant (SD: 8.43 \pm 3.69 ; SHR: 9. 33프.
\end{abstract}

Conclusion: DDFPe treatment provides significant neuroprotection when assessed six hours post stroke.

Keywords: Animal Model, Dodecafluoropentane Emulsion (DDFPe), Middle Cerebral Artery Occlusion (MCAO), Neuroprotectants, Rat, Stroke.

\section{INTRODUCTION}

Diagnosis and treatment of acute stroke remains a complex problem of enormous magnitude afflicting almost 800,000 Americans each year and generating more long term health costs than any other single illness [1]. Over $98 \%$ of patients with ischemic strokes do not directly benefit from specific hyperacute or acute stroke therapy [2]. Defined post stroke periods in humans range from 0-6 hours (hyperacute), 6-24 hours (acute), 1-7 days (subacute), and 7 days to years (chronic) [3]. Treatment of ischemic but not yet infarcted brain tissue, the penumbra, in the hyperacute period may prevent many of the symptomatic effects seen later in ischemic strokes. Considering the sensitivity of neurons to hypoxic insult, the most beneficial therapeutic window for minimizing neurological deficit from stroke is the hyperacute period. Human long-term outcomes are reliant on therapies in this critical period, hence the adage "time is brain".

*Address correspondence to this author at the Department of Radiology, UAMS, 4300 W. Markham St. Little Rock, AR, 72205 USA;

Tel: 501-686-6910; Fax: 501-686-6910; E-mail: culpwilliamc@uams.edu
Treating stroke is a race against the clock, and the only approved simple human therapy, IV tPA, must be given very promptly to provide a positive effect. At one hour after symptom onset, the odds of therapeutic success are excellent but become negligible within the first 4.5 hours with risks outweighing the rewards beyond this point. More complex therapies, intra-arterial interventions, are more difficult to perform quickly and the probability of a successful outcome also diminishes rapidly over time. The solution must be some sort of neuroprotective therapy, a method of stopping the clock on brain death, at least long enough for thrombolysis or embolism extraction to be accomplished. No neuroprotection method has yet reached general clinical use. Dodecafluoropentane emulsion (DDFPe) provides the opportunity to prolong the intervention window, providing longer treatment opportunity and thus the potential for an increased rate of favorable outcomes. This ability of DDFPe administration to "stop the clock" is attributed to its superior properties as a perfluorocarbon oxygen carrier and has been previously demonstrated in rabbit stroke studies.

DDFPe, a $4^{\text {th }}$ generation neuroprotectant perfluorocarbon was tested as an ultrasound contrast agent in humans; 
however, it was also found to be highly efficient at oxygen and carbon dioxide transportation in vivo $[4,5]$. It is the best of the perfluorocarbons in oxygen transportation, probably due to its transition from liquid to gas phase (where oxygen transport dramatically increases) at $29.5^{\circ} \mathrm{C}$. It was shown in rat, pig, and dog studies to be effective in prolonging life in acute blood loss [6-8], and produced $>80 \%$ reductions in acute infarction following permanent embolic stroke in the rabbit embolic stroke model $[9,10]$. Repeated application of DDFPe in the rabbit over a 24 hour period markedly reduced stroke as measured by histologic and vital stains.

No neuroprotection technique has yet been accepted into routine clinical care. Bringing DDFPe to human trials requires a second animal model. Therefore, we chose to test DDFPe in the well-developed rat permanent stroke model with its superior system of neurological tests.

The rat middle cerebral artery occlusion (MCAO) model includes several surgical variations that have resulted in reproducible outcomes including excellent functional measurements and extent of infarction measurements [11]. Variability of outcomes is commonly encountered in different rat strains, different laboratories, and over time in both permanent and temporary MCAO models [7]. Costs, therapeutic sensitivity, and other factors impact the choice of rat strain for experimentation. Spontaneous Hypertensive Rats (SHR) often require more therapeutic effect than Sprague Dawley (SD) rats to demonstrate valid changes. This study measured the percent stroke volume (\%SV) and detailed behavioral outcomes (NAS) in the critical 6 hour hyperacute period following a permanent unilateral MCAO in two strains of rats, the SD and the SHR.

\section{MATERIALS AND METHODOLOGY}

All procedures were approved by the University of Arkansas for Medical Sciences IACUC and are in accordance with the standards set forth in the eighth edition of the Guide for the Care and Use of Laboratory Animals (National Academy of Sciences, The National Academies Press, Washington, D.C.). A total of $\mathrm{n}=26 \mathrm{SD}$ (male, $300 \mathrm{~g}$ ) and $\mathrm{n}=14$ SHR (male, 250-300 g) were used (Harlan Laboratories, St. Louis, MO, USA). In addition nine SHR were used in initially optimizing the stroke model, and five SHR were discarded from the $4 \mathrm{x}$ DDFPe group due to technical difficulties; these were not included in the group numbers. The NAS measurement failed to be obtained on one SD control animal, but this animal is included in evaluation of \%SV. Rats were anesthetized using isoflurane, initiation $3-4 \%$ and maintenance $1-1.5 \%$ in $\mathrm{O}_{2}$ at $1 \mathrm{~L} / \mathrm{min}$. Body temperature was maintained at $37.5 \pm 0.5^{\circ} \mathrm{C}$.

\section{MCAO Surgery}

The permanent MCAO model is a variation of the rat stroke model procedure and surgeries described by Tamura [12], Bederson et al. [13] and Brint et al. [14]. In brief, after a lateral craniotomy and incision of the dura mater, surgical exposure and cauterization of the middle cerebral artery and ipsilateral ligation of the common carotid artery was performed under anesthesia as above. After surgery the rats were allowed to recover.

\section{Groups}

MCAO rats were randomly placed in one of three groups; 1) control (saline diluent), or 2) one dose of $0.6 \mathrm{ml} / \mathrm{kg}(1 \mathrm{x}$ DDFPe) of $2 \%$ DDFPe (NuvOx Pharma; Tucson, AZ, USA), or 3) four doses of $0.6 \mathrm{ml} / \mathrm{kg}$ DDFPe (4x DDFPe) at 90 minute intervals. All groups had tail vein or jugular vein IV catheters in place for administration of DDFPe or saline diluent and were given heparinized saline to prevent blockage of catheters. There were $\mathrm{n}=12,7$ and $7 \mathrm{SD}$ rats; and $\mathrm{n}=7,3$ and 4 SHR rats, in the respective treatment groups. DDFPe was started at one hour following MCAO and repeated every 90 minutes for a total of 1 or 4 doses.

\section{Neurological Assessment}

The neurological assessment score (NAS) evaluation of animals after inducement of stroke provides a measure of behavioral deficit severity. The extent of ischemic injury to rat brain is frequently reported using the rat NAS [15] (Table 1). The total score is a composite of motor, sensory, reflex, and balance tests and ranges from 0 (normal score) to 18 (maximal deficit score). Scores of 1-7 are considered mild, 8-12 moderate and 13-18 severe. Rats were tested for neurological deficits immediately prior to euthanasia. NAS values are used for comparison among the three treatment groups.

\section{Euthanasia}

Rats were euthanized by placing them into an empty container that then received $\mathrm{CO}_{2}$ from a tank source with a flow rate displacing up to $30 \%$ of the chamber volume per $\min$ for 4 min [16]. For a confirmation of death, a bilateral pneumothorax was performed.

\section{TTC Staining and Stroke Infarct Volume Measurement}

After each rat was euthanized the brain was removed, cut into $0.2 \mathrm{~cm}$ thick sections, and stained at $37^{\circ} \mathrm{C}$ for 1 hour with the vital stain triphenyl tetrazolium chloride (TTC). TTC-stained sections were photographed and image analysis software was used to determine the stroke volume as a percent of total brain volume.

\section{Statistics.}

Summary statistics for $\% \mathrm{SV}$ and NAS are presented as mean \pm standard deviation. The influence of treatment and rat strain on \%SV and NAS assessments were evaluated in general linear models using PROC GLM in SAS v. 9.3 (SAS Institute Inc., Cary, NC). Because of the unequal sample sizes, group differences were tested using the least squares means option in that procedure. The reported group comparisons were pre-planned and the p-values reported are not adjusted for multiple testing.

\section{RESULTS}

\section{Percent Stroke volume}

Since neither the strain nor the treatment-by-strain interaction terms were significant in the general linear model, $(\mathrm{P}=0.93, \mathrm{P}=0.70$ respectively) $\% \mathrm{SV}$ was compared 
Table 1. Neurological assessment score points.

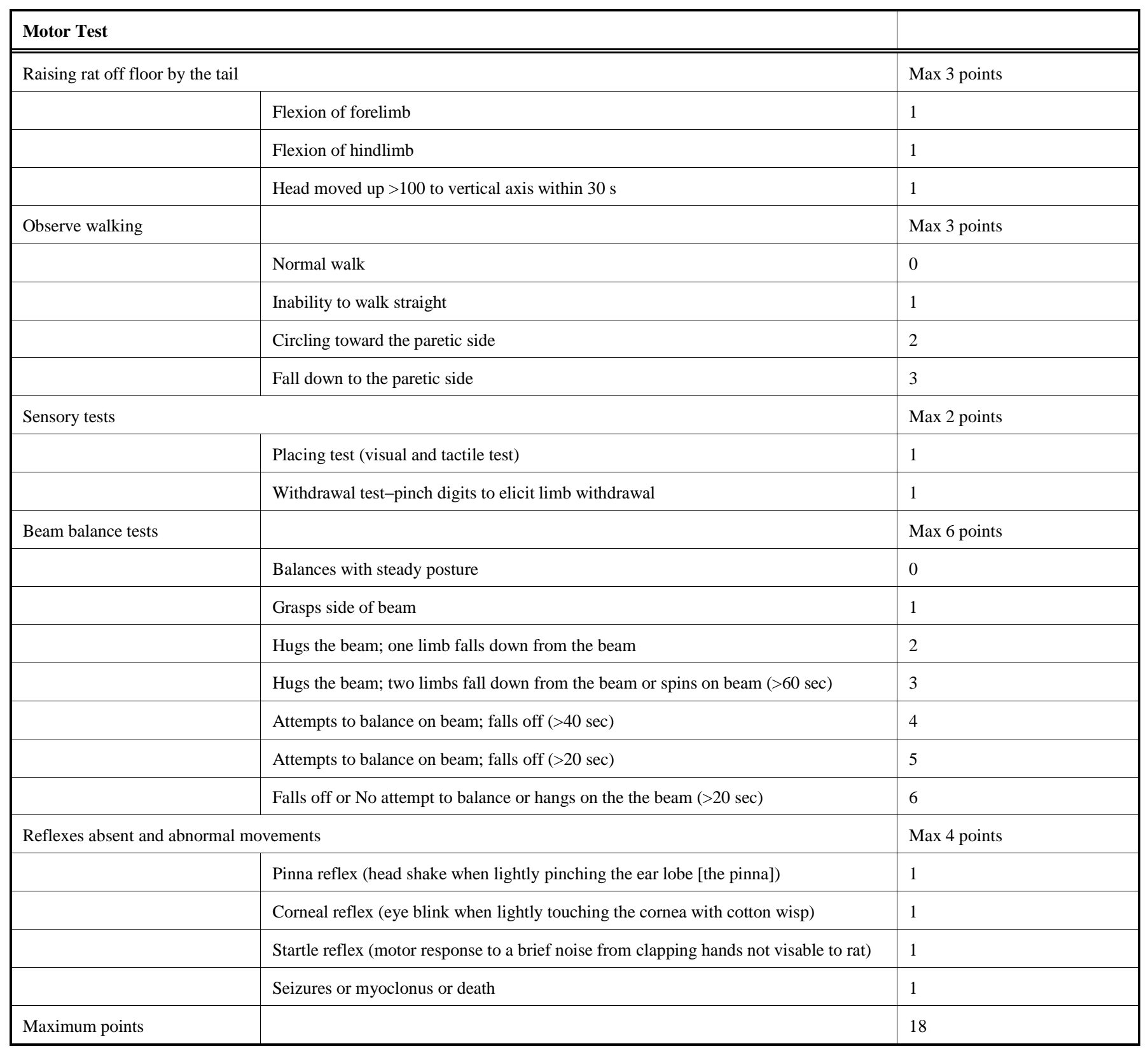

across experimental groups without further consideration of strain. DDFPe significantly decreased $\% \mathrm{SV}$ in the $1 \mathrm{x}$ and $4 \mathrm{xDDFPe}$ groups compared to the control group $(2.59 \pm 1.81$ and $0.98 \pm 0.88$ vs. $9.24 \pm 6.06$, at $\mathrm{p} \leq 0.001$ in each case; $\mathrm{p}<0.0001$ for the overall test for treatment effect) (Fig 1A). The $1 \mathrm{x}$ versus the $4 \mathrm{xDDFPe} \mathrm{SD}$ groups were not significantly different $(\mathrm{p}=0.40)$.

\section{Neurological Assessment}

Both strain and treatment were significant factors in a general linear model of NAS which did not include a nonsignificant interaction term. Therefore, groups were compared by strain-treatment combination. Within both the SD and SHR strains, unadjusted comparisons of leastsquares means revealed significant differences between the
4xDDFPe group and controls (SD: $5.00 \pm 2.45$ vs. $9.36 \pm 3.56$, $\mathrm{p}=0.01$; SHR: $7.75 \pm 4.43$ vs. 12.14 $\pm 3.08, \mathrm{p}=0.05)$, but differences between the $1 \mathrm{xDDFPe}$ group and controls were not significant (1x DDFPe values - SD: 8.43+3.69; SHR: 9.33+3.51) (Fig 1B). The $1 \times$ DDFPe and 4xDDFPe groups did not differ significantly in either strain. Although strain was a significant factor in the overall model, none of the strain comparisons within treatment groups were significant, likely due to small sample sizes.

\section{Correlation of \% Stroke Volume vs. Neurological Assessment}

As depicted in Fig. (2), a positive correlation exists between \%SV and NAS score. Analysis of covariance indicates the level of correlation differs significantly among 

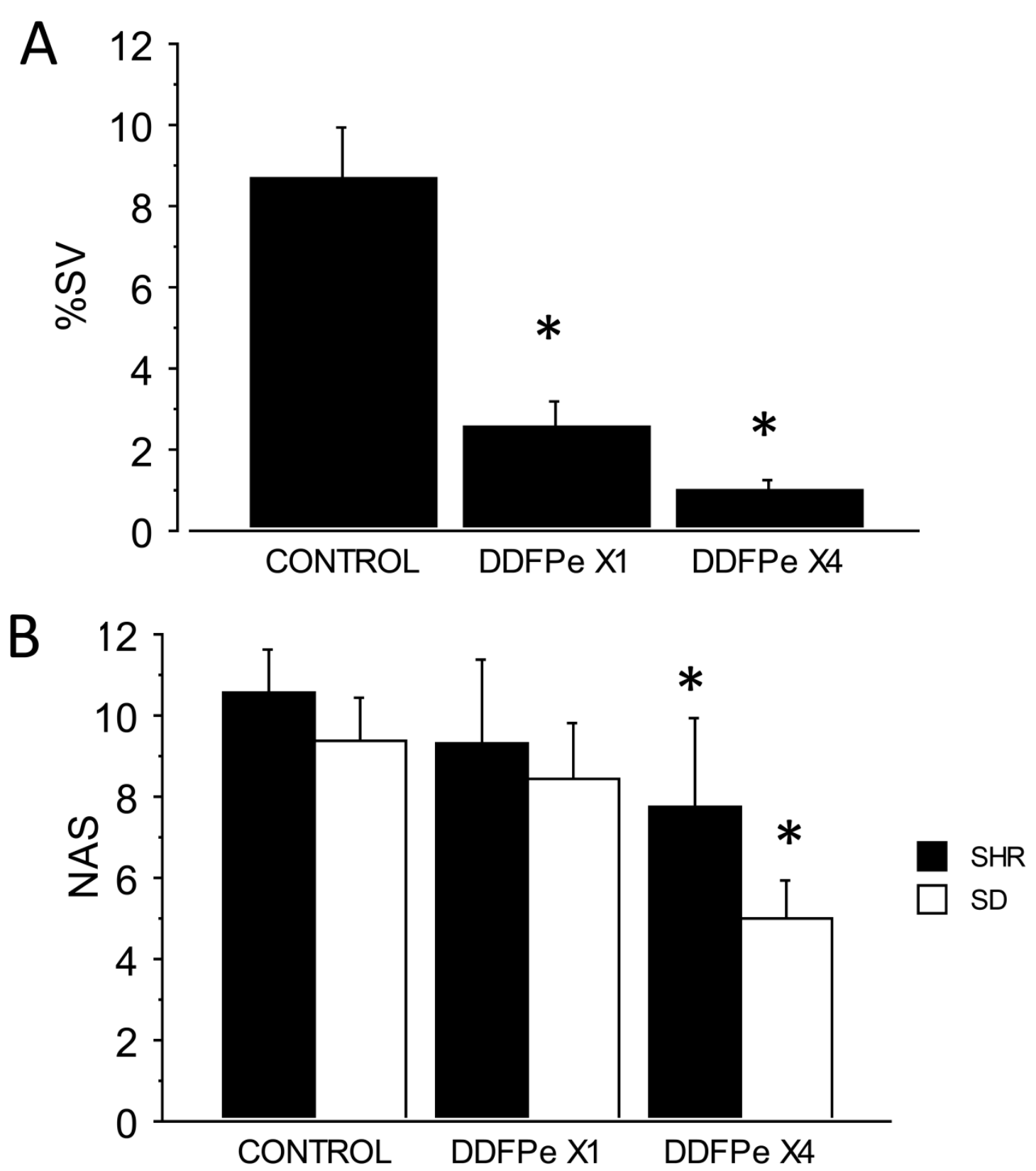

Fig. (1). Bar chart of $\% \mathrm{SV}$ and NAS data.

A. Percent stroke volume $(\% \mathrm{SV})$ in rats 6 hours after DDFPe treatment. Rats had significantly lower \%SV with DDFPe treatment at $1 \mathrm{x}$ and $4 \mathrm{x}$ treatment vs. controls at $* \mathrm{p} \leq 0.001$. Means are \pm SD.

B. Neurological function scores (NAS) in SHR and SD male rats 6 hours after DDFPe treatment. SHR (solid bar) and SD (open bar) rats treated with $4 \mathrm{x}$ DDFPe had improved neurological function vs. controls $* \mathrm{p}=0.05$. Both SHR and SD $1 \mathrm{x}$ DDFPe $v s$. controls were $\mathrm{p}=\mathrm{NS}$. Means are \pm SD.

the three treatment groups $(\mathrm{p}=0.002)$. Further analysis indicated the correlation did not differ significantly between the two groups receiving DDFPe $(\mathrm{p}=0.89)$. The correlation in the combined DDFPe groups $(\mathrm{r}=0.47)$ differs significantly from that in the control animals $(r=0.75) ; p=0.0005$.

\section{DISCUSSION}

In this study a marked tissue saving action was found for DDFPe therapy in two strains of rats as determined at six hours post stroke. Both percent stroke volume (\%SV) and neurological test scores (NAS) were markedly reduced.
Thus, DDFPe provides effective neuroprotection in the critical hyperacute phase by reducing the infarct and improving neurological test scores.

This corroborates and expands previous rabbit experiments that found DDFPe dramatically reduced infarct volume in the hyperacute phase at four hours post-occlusion [9] and that repeated dosing extended the benefit through the acute phase to the sub-acute phase beginning 24 hours following permanent arterial occlusion [10]. The reduction in infarct volume demonstrates that DDFPe preserves vital tissue despite diminished blood flow and without 


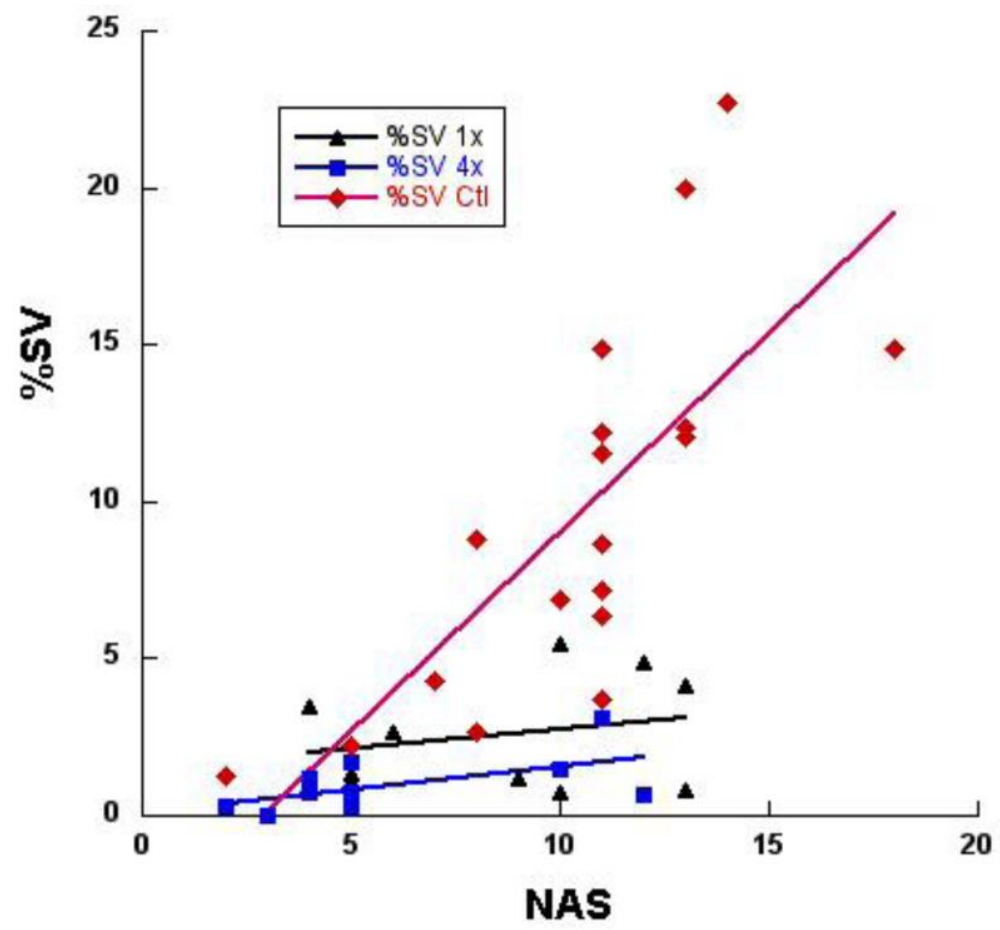

Fig. (2). Regression analysis of \%SV correlated with neurological assessment scores (NAS). Combined data of both SD and SHR rats $(\mathrm{n}=39)$, in the controls $(\diamond)$ indicate increasing $\% \mathrm{SV}$ was associated with a positive correlation of increasing deficits at $\mathrm{r}=0.75$, in the $1 \mathrm{x}$ DDFPe (symbol) correlation $r=0.24$, and in the $4 \mathrm{x}$ DDFPe $(\boldsymbol{a})$ correlation $\mathrm{r}=0.58$, both significantly different from control, $\mathrm{p} \leq 0.01$. This suggests a therapeutic effect of DDFPe.

reperfusion. Further testing in a second animal model was desirable in order to confirm the tissue-preserving action of DDFPe, and to study possible DDFPe therapeutic preservation of neurological function.

The rat was chosen for this study because of its relatively consistent stroke size and location in the middle cerebral artery occlusion (MCAO) model as well as the excellent behavioral assessment system available in rats. The rat has a larger repertoire of testable behaviors than does the rabbit, enhancing the outcome evaluation in this model. In addition to the frequently used and less expensive Sprague-Dawley rat strain, the Spontaneously Hypertensive rat was used because of its reported higher rate of success for inducement of stroke in the MCAO model [17].

Our rat studies replicated the excellent tissue sparing previously reported in rabbits in the hyperacute phase. Further, the neurological function at six hours was also markedly improved with repeated DDFPe dose regimens. Rats receiving only one dose did not show improvement in NAS values suggesting that the beneficial effect lasts less than the five hours from administration to NAS measurement. However, the TTC findings were moderately improved with only one dose suggesting that these tissue changes may begin only after the effect of DDFPe slowly wears off. It also suggests that the NAS deficit may still be reversible with repeated doses.

We speculate that the $4 x D D F P e$ treatment preserved more neurons within the penumbra over the five hour period than the $1 x$ treatment. This could preserve more neural network connections and thus account for the better NAS values. Although the $1 \times$ DDFPe treatment reduced stroke volume measured by metabolism on TTC, it is apparent that damaged neurons often did not function well on NAS. A slight increase in neuronal death in the $1 \mathrm{x}$ treated rats over that in the $4 \mathrm{x}$ treated rats could result in critical interruptions of neural networks responsible for the brain functions assessed by the NAS values. Perhaps the TTC measures can be supplemented with a more sophisticated technique such as fluoro-jade staining [18] in the future.

It is well proven that MRI and CT images of human stroke at initial diagnosis show infarct volumes closely correlate to eventual clinical outcomes based on neurological function at 90 days $[19,20]$. In this rat study infarct volumes and neurological outcomes show significant correlation in the control population, Figure 2. As \%SV increases the NAS also increases. However, in the DDFPe therapy groups the correlation is much less impressive. While some animals did show increased NAS values there was much less increase in $\% \mathrm{SV}$. A different relationship must apply here and produce less acute tissue metabolic effect. The affected brain may be damaged and not functioning as measured on NAS but not yet dead, therefore not seen as a TTC deficit. This area might or might not recover. More study is required to clarify this.

In the clinical race to reperfuse ischemic brain the limited time window excludes many patients from receiving treatment. No neuroprotective technique is yet in general use, but DDFPe provides the opportunity to prolong the intervention window, providing greater treatment 
opportunity and the possibility of increased favorable outcomes. This "stop the clock" consequence of DDFPe administration is attributed to its well proven properties as a gas transporter.

The utility of DDFPe in the setting of stroke lies in its ability to carry oxygen. In vivo, it is thought that DDFPe forms nanobubbles or perhaps even a "plasma-like interface" [8]. There is some speculation that DDFPe may accumulate in ischemic tissues and participate in transport of oxygen through a serial transport mechanism. These nanodroplets do seem to diffuse quickly through tissues (unpublished observation) and also penetrate thrombi, making DDFPe an ideal neuroprotectant in the face of embolus. Furthermore, the small size of DDFPe permits delivery of oxygen beyond erythrocyte accessibility. Regardless of mechanism, DDFPe displays extraordinary oxygen carrying ability and has been shown in an anemic rat model to raise oxygen saturation 40$100 \%$ [6] and compensate for $90 \%$ of the oxygen deficiency [7]. Such oxygen carrying abilities would be beneficial in the context of acute ischemic stroke. Since hypoxia stands as one of the major elicitors of stroke damage, enhancing oxygen content in the blood and critical tissues offers potential to rescue brain tissue in the penumbra.

This study is limited by the short times tested. This hyperacute phase is extremely important, certainly the most critical of all, but needs to be expanded, with reperfusion, into the acute, sub-acute and chronic phases. Reperfusion can be problematic in some rat models, but preservation of final neurological function with reperfusion would militate strongly for progressing to human trials. Small sample size also limits some categories where attrition reduced desired numbers.

\section{CONCLUSION}

In summary, the rat model shows dramatic improvement in $\% \mathrm{SV}$ and NAS with multiple doses of IV DDFPe for a six hour period following permanent MCA occlusion. This indicates that DDFPe indeed has an effect in reducing infarct and providing neuroprotection during the critical six hour hyperacute period. Further animal study is justified with the goal of rapid translation into human trials.

\section{ABBREVIATIONS}

$\begin{array}{lll}\text { DDFPe } & & \text { Dodecafluoropentane emulsion } \\ \text { MCAO } & & \text { middle cerebral artery occlusion } \\ \text { NAS } & \text { neurological assessment scores } \\ \% \mathrm{SV}= & \text { percent stroke volume } \\ \mathrm{SD}= & \text { Sprague Dawley } \\ \mathrm{SHR}= & \text { Spontaneously Hypertensive Rats } \\ \mathrm{TTC}= & \text { triphenyl tetrazolium chloride }\end{array}$

\section{CONFLICT OF INTEREST}

Drs. Culp and Skinner have a use patent pending on DDFPe in stroke.

\section{ACKNOWLEDGEMENTS}

Shaoke Luo MD, Kelly Ball and John Lowery DVM are acknowledged for participation. The DDFPe was kindly provided by NuvOx Pharma, Tucson, AZ. This study was self-funded.

\section{REFERENCES}

[1] Ferris A, Robertson R, Fabunmi R, Mosca L. American heart association and american stroke association national survey of stroke awareness among women. Circulation 2005; 111: 1321-26.

[2] Go A, Mozaffarian D, Roger V, et al. Heart Disease and Stroke Statistics - 2014 Update: A Report from the American Heart Association. Circulation Online December 18, 2013. Print ISSN: 0009-7322. Online ISSN: 1524-4539.

[3] Xavier R, Qureshi A, Kirmani J, Yahia A, Bakshi R. Neuroimaging of stroke. South Med J 2003; 96: 367-69.

[4] Johnson JLH, Dolezal MC, Kerschen A, Matsunaga TO, Unger EC. In vitro comparison of dodecafluoropentane (DDFP), perfluorodecalin (PFD), and perfluoroctylbromide (PFOB) in the facilitation of oxygen exchange. Artif Cell Blood Substit 2009; 37: 156-162.

[5] Riess JG. Understanding the fundamentals of perfluorocarbons and perfluorocarbon emulsions relevant to in vivo oxygen delivery. Artif Cell Blood Substit 2005; 33: 47-63.

[6] Lundgren C, Bergoe G, Olszowka A, Tyssebotn I. Intravascular fluorocarbon-stabilized microbubbles protect against fatal anemia in rats. Artif Cell Blood Substit 2006; 34: 473-68.

[7] Lundgren C, Bergoe G, Olszowka A, Tyssebotn I. Tissue nitrogen elimination in oxygen-breathing pigs is enhanced by fluorocarbonderived intravascular micr-bubbles. UHM 2005; 32: 215-26.

[8] Johnson EC, Erickson BK, Podolsky A, Birks EK, Keipert PE, Faithfull NS, Wagner PD. Effects of a perfluorocarbon emulsion for enhanced $\mathrm{O} 2$ solubility on hemodynamics and $\mathrm{O} 2$ transport in dogs. J Appl Physiol 1995; 79: 1777-86.

[9] Culp WC, Woods SD, Skinner RD, Brown AT, Lowery JD, Johnson JLH, Unger EC, Hennings LJ, Borrelli MJ, Roberson PK. Dodecafluoropentane emulsion decreases infarct volume in a rabbit ischemic stroke model. JVIR 2012; 23: 116-21.

[10] Woods SD, Skinner RD, Ricca AM, Brown AT, Lowery JD, Borrelli MJ, Lay JO, Culp WC. Progress in dodecafluoropentane emulsion as a neuroprotective agent in a rabbit stroke model. Mol Neurobiol 2013; 48: 363-7.

[11] Aspey BS, Taylor FL, Terruli M, Harrison MJ. Temporary middle cerebral artery occlusion in the rat: consistent protocol for a model of stroke and reperfusion. Neuropathol Appl Neurobiol 2000; 26: 232-42.

[12] Tamura A, Graham DI, McCullough J, Teasdale GM. Foca cerebral ischemia in the rat: 1 . Description of technique and early neuropathological consequences following middle cerebral artery occlusion. J Cereb Blood Flow Metab 1981; 1: 53-60.

[13] Bederson JB, Pitts LH, Tsuji M, Nishimura MC, Davis RL, Bartkowski H. Rat middle cerebral artery occlusion: evaluation of the model and development of a neurologic examination. Stroke 1988; 17: 472-6.

Brint S, Jacewicz M, Kiessling M, Tanabe J, Pulsinelli W. Focal brain ischemia in the rat: methods for reproducible neocortical infarction using tandem occlusion of the distal middle cerebral and ipsilateral common carotid arteries. J Cereb Blood Flow Metab 1988; 8: 474-85.

Zhou J, Zhuang J, Li J, et al. Long-term post-stroke changes include myelin loss, specific deficits in sensory and motor behaviors and complex cognitive impairment detected using active place avoidance. PLoS One 2013; 8: e57503.

Chen J, Li Y, Wang L, Lu M, Zhange X, Chopp M. Therapeutic benefit of Intracerebral transplantation of bone marrow stromal cells after cerebral ischemia in rats. J Neurol Sci 2001; 189: 49-57. Ren Y, Hashimoto M, Pulsinelli WA, Nowak TS. Hypothermic protection in rat focal ischemia models: strain differences and relevance to "reperfusion injury". J Cerebral Blood Flow Metab 2004; 24: 42-53.

[18] Liu F, Schafer DP, McCullough LD. TTC, Fluoro-Jade B and NeuN staining confirm evolving phases of infarction induced by 
middle cerebral artery occlusion. J Neurosci Method 2009; 179: 18.

[19] Vogt G, Laage R, Shuaib A, Schneider A. Initial lesion volume is an independent predictor of clinical stroke outcome at day 90 . Stroke 2012; 43: 1266-72.
[20] Yoo AJ, Chaudhury ZA, Nogueira RG, Lev MH, Schaefer PW. Infarct volume is a pivotal biomarker after intra-arterial stroke therapy. Stroke 2012; 43: 1323-30.

Received: September 10, 2014

Revised: October 24, 2014

Accepted: October 25, 2014

(C) Brown et al.; Licensee Bentham Open.

This is an open access article licensed under the terms of the Creative Commons Attribution Non-Commercial License (http://creativecommons.org/licenses/ by-nc/3.0/) which permits unrestricted, non-commercial use, distribution and reproduction in any medium, provided the work is properly cited. 